\title{
Single-top t-channel production with off-shell and non-resonant effects
}

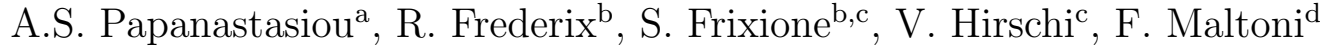 \\ ${ }^{a}$ DESY, Deutsches Elektronen-Synchrotron, Notkestraße 85, \\ D-22607 Hamburg, Germany \\ ${ }^{b}$ PH Department, TH Unit, CERN, CH-1211 Geneva 23, Switzerland \\ ${ }^{c}$ ITPP, EPFL, CH-1015 Lausanne, Switzerland \\ ${ }^{d}$ Centre for Cosmology, Particle Physics and Phenomenology (CP3), \\ Université catholique de Louvain, B-1348 Louvain-la-Neuve, Belgium
}

\begin{abstract}
This letter details and discusses the next-to-leading order QCD corrections to $t$-channel electro-weak $W^{+} b j$ production, where finite top-width effects are consistently taken into account. The computation is done within the AMC@NLO framework and includes both resonant and non-resonant contributions as well as interferences between the two. Results are presented for the LHC and compared to those of the narrow-width approximation and effective theory approaches.
\end{abstract}

Keywords: Top quark, finite width effects, LHC

\section{Introduction}

Since its observation at the Tevatron in 2009 [1, 2], single-top production has played an important role in top-quark phenomenology at hadron colliders despite having a smaller cross section than that of $t \bar{t}$. This channel is not only an important background to other processes, such as top-pair and Higgs production, but it is also interesting in its own right, for example for its potential to allow a direct determination of the CKM matrix element $V_{t b}$ (see [3] for a recent discussion on this point), as well as being sensitive to new physics effects in many beyondthe-Standard Model scenarios. Single-top production is also a process through which the measurements of key top quark properties such as the mass, $m_{t}$, can be performed independently from those in $t \bar{t}$ production. This is particularly useful because the majority of the systematic uncertainties are expected to be fairly different in these two processes. 
It is of no surprise therefore that over the last three decades much effort has been invested in providing accurate predictions for single-top cross sections. Stable single-top production was first discussed at the leading order (LO) in [4]. Next-to-leading order (NLO) QCD corrections in the five-flavour (5F) scheme were first computed in [5] and in the four-flavour $(4 \mathrm{~F})$ scheme in $[6,7]$, whilst electroweak (EW) corrections were investigated in [8]. Furthermore, soft and collinear gluon resummation has been studied in $[9,10,11,12]$ and stable single-top at NLO matched to parton showers has been achieved in $[13,14,15]$ for both the $5 \mathrm{~F}$ and $4 \mathrm{~F}$ schemes. Incorporating the decay of the top quark, $t \rightarrow W^{+} b$, as part of the hard process matrix elements was accomplished via the narrow-width approximation (NWA) in $[16,17,18]$, in which NLO corrections to both production and decay were included. Going beyond the NWA, a study of off-shell and non-resonant effects at LO was performed in [19] and final-state non-factorizable corrections in the $s$-channel were examined in [20]. In [21, 22], effective theory (ET) techniques were employed to relax the assumption of an on-shell top quark in the amplitudes, allowing for a systematic study of finite top-width $\left(\Gamma_{t}\right)$ effects in the resonant regions of phase space.

The full computation of $\alpha_{s}$-corrections to $\mathrm{EW} W^{+} b j$ production (i.e. the process that includes single-top production) has so far been missing in the literature. Given the opportunity for precision top physics provided by the LHC, it is of phenomenological interest to understand the effects of off-shell top quarks versus top quarks in the NWA. In this letter we present such a computation for the $t$ channel process in the $5 \mathrm{~F}$ scheme, and make a direct comparison to results in the NWA as well as to those obtained by using the ET method. The $s$-channel and $W t$-production processes are not considered here.

The expectation is that for inclusive observables off-shell effects are small $[23,24,25]$ (i.e., parametrically of $\mathcal{O}\left(\Gamma_{t} / m_{t}\right)$ ), whilst they should be noticeable in the case of less inclusive observables, such as the invariant or transverse masses of the reconstructed top. Indeed, differences between NWA and ET or off-shell calculations have already been highlighted for $5 \mathrm{~F}$ scheme single-top [21, 22] and top-pair [26, 27, 28, 29] production. We shall confirm these findings here.

This paper is organized as follows. In Sect. 2 we briefly discuss the NWA, $\mathrm{ET}$, and off-shell approaches in view of their application to single-top production; results for total rates and for a few selected distributions are presented in Sect. 3; we conclude in Sect. 4. 


\section{Unstable single-top production}

In the NWA limit $\Gamma_{t} \rightarrow 0$ the following replacement is made in the squared amplitude,

$$
\frac{1}{\left(p_{t}^{2}-m_{t}^{2}\right)^{2}+\Gamma_{t}^{2} m_{t}^{2}} \longrightarrow \frac{\pi}{m_{t} \Gamma_{t}} \delta\left(p_{t}^{2}-m_{t}^{2}\right)
$$

The error introduced by making this approximation is expected to be of order $\Gamma_{t} / m_{t}$. The replacement above leads to an exact factorization of the matrix elements into terms describing the production and the decay of on-shell top quarks. This factorization can be combined with a strict expansion of $\Gamma_{t}$ in $\alpha_{s}$ to yield results correct to $\mathcal{O}\left(\alpha_{s}\right)[30,31]$.

Maintaining a finite width and subsequently relaxing the on-shell assumption in standard fixed-order perturbation theory entails the computation of much more than the resonant diagrams (i.e., those that feature an $s$-channel top-quark propagator) required in the NWA. Namely, at any given perturbative order one should include all the resonant and non-resonant diagrams (the latter feature a $W$-boson and a $b$ quark, which are not connected to each other in a $t W b$ vertex) in order not to break gauge invariance.

In the region where the invariant mass of the $W b$ pair is close, but not necessarily equal to $m_{t}$, the cross-section is dominated by resonant diagrams. It is possible to make use of this fact to construct an expansion of the matrix elements around the pole of the top-quark propagator [32, 33]. The pole expansion has been generalized through the use of ET techniques in [21, 22, 29], where applications to the processes of single-top and top-pair production were also made, thus capturing the dominant finite-width effects.

The disadvantage of both the NWA and ET approaches is that they are in principle only valid in regions of phase space where the top quarks are either on or near their mass-shell. A gauge-invariant way of introducing a finite-width whilst ensuring that the final result is valid over the full phase space is the complex-mass scheme (CMS) [34, 35], whereby the top-quark width is consistently included at the level of the Lagrangian via the procedure of renormalization. The CMS is a generalization of the on-shell renormalization scheme and has been recently employed by two groups to perform NLO calculations of unstable top-pair production $[26,27,28]$. For the case of unstable top quarks, it amounts to expressing the bare mass in terms of a complex renormalized mass and a complex counter-term,

$$
m_{t, 0}=\mu_{t}+\delta \mu_{t}
$$




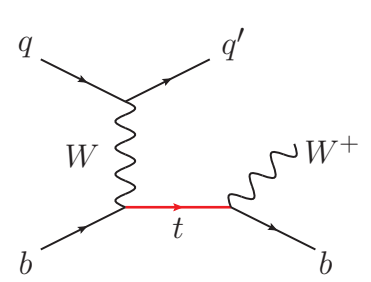

(a)

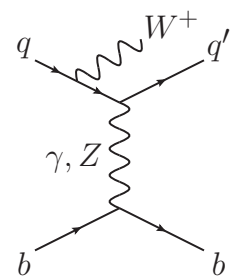

(b)

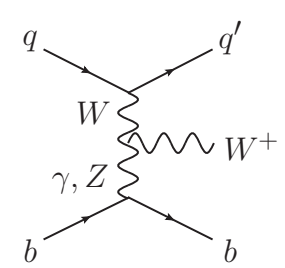

(c)

Figure 1: Selection of LO $t$-channel diagrams for $\mathrm{EW} W^{+} b j$ production in the $5 \mathrm{~F}$ scheme: resonant (a) and non-resonant (b) \& (c).

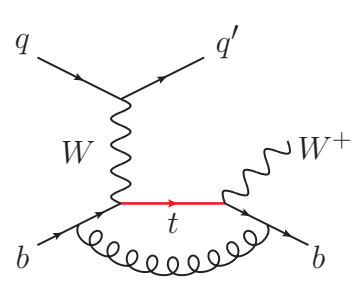

(a)

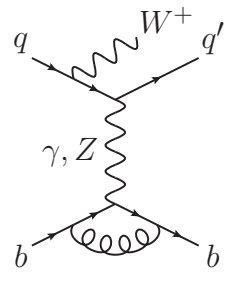

(b)

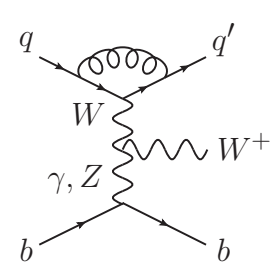

(c)

Figure 2: Selection of NLO virtual $t$-channel diagrams for $\mathrm{EW} W^{+} b j$ production in the $5 \mathrm{~F}$ scheme: resonant (a) and non-resonant (b) \& (c).

where $\mu_{t}^{2}=m_{t}^{2}-i m_{t} \Gamma_{t}$. As is shown explicitly in [35], the quantity $\delta \mu_{t}$ can be fixed in terms of the renormalized top-quark self-energy evaluated at the complex argument, $p_{t}^{2}=\mu_{t}^{2}$, such that $\mu_{t}^{2}$ corresponds to the complex pole of the top quark propagator. The precise value of the top width can be freely chosen as an input in this scheme; but in order to ensure NLO accuracy, the width correct to (at least) $\mathcal{O}\left(\alpha_{s}\right)$ should be used.

The CMS has recently been implemented [36] in the framework of AMC@NLO, and the results presented in this paper illustrate the first hadron-collider application of this new feature. The automation of such an approach to unstable particle production and decay is highly beneficial due to the non-trivial book-keeping involved in these calculations. The NLO corrections have thus been obtained in an automated fashion, with the one-loop and real contributions computed using MADLOOP [37] and MADFKS [38] respectively.

\subsection{Process definition}

Given that our aim is that of comparing the predictions of the NWA and ET approaches to single-top production with those we obtain by retaining all $\Gamma_{t}$ and 


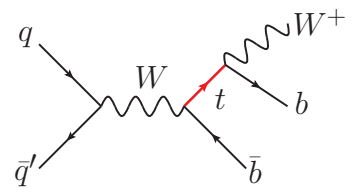

(a)

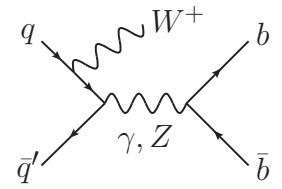

(b)

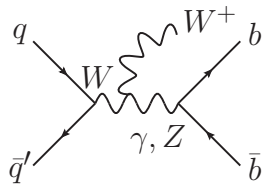

(c)

Figure 3: Selection of LO $s$-channel diagrams for $\mathrm{EW} W^{+} b j$ production: resonant (a) and non-resonant (b) \& (c). These are not included in our calculation.

interference effects, the process we consider is:

$$
p p \rightarrow W^{+} J_{b} J_{\text {light }}+X .
$$

$J_{b}$ is defined to be a jet that contains at least a $b$ quark, while $J_{\text {light }}$ is a jet that may (or may not) contain $b$ or $\bar{b}$ quarks. Furthermore, at the Born level, we only keep contributions to the cross-section of $\mathcal{O}\left(\alpha_{W}^{3}\right)$. NLO QCD corrections to this set of diagrams are then computed, yielding contributions of $\mathcal{O}\left(\alpha_{W}^{3} \alpha_{S}\right)$. We stress that, while this is a straightforward procedure from an algorithmic point of view when the top quark is on-shell, it becomes highly non-trivial when this condition is relaxed. In particular, for the process at hand, the computation of NLO corrections in QCD is well defined only if the CKM matrix is diagonal in the third generation (i.e., $V_{t b}=1$ ). In fact, when $V_{t b} \neq 1$, Born-level amplitudes of $\mathcal{O}\left(g_{W} g_{S}^{2}\right)$ (which feature a gluon propagator) have a non-null interference with those we consider here, thus resulting in a contribution of $\mathcal{O}\left(\alpha_{W}^{2} \alpha_{S}\right)$ to the Born cross-section. The EW corrections to this term are therefore of $\mathcal{O}\left(\alpha_{W}^{3} \alpha_{S}\right)$, which is the same order as the NLO QCD corrections to pure-EW Born matrix elements. One would therefore have no choice but to compute both types of correction ${ }^{1}$. In order to avoid such complications we will not consider the case $V_{t b} \neq 1$ any further.

A consequence of having a third-generation diagonal CKM is that, up to NLO in QCD and in the 5F scheme, $t$ - and $s$-channel contributions to $W b j$ production do not interfere (note that this is true in the case of on-shell top production regardless of the nature of the CKM matrix). We can thus safely adopt the procedure of simply excluding $s$-channel Feynman diagrams, keeping only those that feature the exchange of $t$-channel EW vector bosons (samples of the latter

\footnotetext{
${ }^{1}$ Having said that, we note that these interference terms are strongly CKM-suppressed, since one needs two off-diagonal CKM matrix elements that connect a $b$ with a first- or secondgeneration quark.
} 
diagrams are depicted in Figures 1 and 2, and of the former diagrams in Figure 3). In fact, at the LO the identities of the partons in $t$-channel diagrams can never be equal to those entering $s$-channel ones. This is not the case at the NLO, where the two channels can however be simply distinguished by looking at their colour structures. Discarding $s$-channel diagrams has the advantage of allowing us to directly compare with $t$-channel results available in the literature.

Even when setting $V_{t b}=1$, there is another important difference with respect to the case of an on-shell top quark. Namely, while the latter does not need any Born-level kinematic cuts to be well defined, this is not the case for the full offshell process, because of the presence of potentially singular contributions from non-resonant diagrams - see for example Figure 1b, where the photon propagator diverges when the outgoing $b$ quark is parallel to the beam line. This implies that it is necessary to impose a constraint on the final-state $b$-jet for our CMS computation to be well defined. We can summarise the discussion so far as follows: when $V_{t b}=1$ the $t$-channel contribution to the process of Eq. (3) and its NLO QCD corrections are finite and well defined, provided that $J_{b}$ has a non-zero transverse momentum.

While the $s$-channel cross section is strongly suppressed at the LHC, one may wonder whether it can be competitive with the non-resonant effects in the $t$ channel. Although we shall not study this problem here, we note that it is possible to include both $s$ and $t$ channels in our approach, provided we slightly modify the definition of $J_{b}$ to being a jet that contains one $b$ quark, but not a $b \bar{b}$ pair (or in other words, $J_{b}$ cannot be $b$-flavour neutral). This definition is in fact sufficient to damp singularities in diagrams that feature a $\gamma \rightarrow b \bar{b}$ splitting (which, being of QED origin, are not subtracted in an NLO QCD computation). As in the previous case, a minimal $p_{T}\left(J_{b}\right)$ must be imposed.

\section{Results}

The minimal conditions necessary to ensure that the process of Eq. (3) is well defined have been given above. In our phenomenological analysis, we supplement these with additional kinematic cuts, which are summarised in Table 1. In this way, similarly to the comparison made recently in [39] between NWA [40, 30, 31] and off-shell $[26,27,28]$ approaches for $t \bar{t}$ production and decay, we ensure not to impose conditions on our final state that could potentially enhance non-resonant contributions. This allows for a fair comparison to be made with the NWA and ET results available in the literature. As far as the NWA is concerned, we use the $t$-channel single-top production and decay process implemented in MCFM 


$$
\begin{array}{cc}
p_{T}\left(J_{b}\right)>25 \mathrm{GeV} & p_{T}\left(J_{\text {light }}\right)>25 \mathrm{GeV} \\
\left|\eta\left(J_{b}\right)\right|<4.5 & \left|\eta\left(J_{\text {light }}\right)\right|<4.5 \\
140 & <M\left(W^{+}, J_{b}\right)<200 \mathrm{GeV}
\end{array}
$$

Table 1: Basic analysis setup allowing for a fair comparison of NWA, ET and off-shell approaches.

$\begin{array}{cc}m_{Z}=91.1876 \mathrm{GeV} & m_{W}=80.3980 \mathrm{GeV} \\ \Gamma_{Z}=2.4952 \mathrm{GeV} & \Gamma_{W}=2.1054 \mathrm{GeV} \\ G_{F}=1.6639 \times 10^{-5} \mathrm{GeV}^{-2} & \alpha_{e}^{-1}=132.3384\end{array}$

Table 2: Input parameters.

$[16,31]$; for the ET approach we have used the $t$-channel single-top code of [22]. In addition to the decay of the top quark, the NWA and ET results we compare to also include the decay $W^{+} \rightarrow e^{+} \nu_{e}$. To ease the comparison we have therefore included a factor of $1 / 9$ in our off-shell results to account for the appropriate $W$ boson branching fraction. However, no information on the decay products of the $W$-boson is used in the analysis we perform.

The pole mass of the top quark is set equal to $m_{t}=173.2 \mathrm{GeV}$ and we use the MSTW2008NLO PDF set [41] (at both LO and NLO), which also provides the value of $\alpha_{s}\left(m_{Z}\right)$. Further input parameters are shown in Table 2. The top quark widths we use at LO and NLO are $\Gamma_{t}^{\mathrm{LO}}=1.5017 \mathrm{GeV}$ and $\Gamma_{t}^{\mathrm{NLO}}\left(\mu=m_{t} / 2\right)=1.3569 \mathrm{GeV}$ respectively. Jets are defined by means of the $k_{t}$-algorithm $[42,43]$, as implemented in FASTJET [44], with $R=0.5$. The central value for the factorization and renormalization scales is $\mu=m_{t} / 2$. These two scales are also simultaneously varied in the range $\mu \in\left[m_{t} / 4, m_{t}\right]$, in order to estimate the size of missing higher order corrections; we point out that while performing these variations, the scale is also consistently changed in the top quark width. The upper panels of the plots we present display the LO (green band) and NLO (blue band) off-shell results, along with the NWA (solid-red with crosses) and ET (solid-magenta with boxes) results. The lower insets show the relative differences between the various approaches (NWA/ET in solid-red/dashed-magenta), by plotting the quantities $d \sigma_{\mathrm{NLO}}^{\mathrm{NWA} / \mathrm{ET}} / d \sigma_{\mathrm{NLO}}^{\text {off-shell }}-1$.

The cross sections at the LO and NLO resulting from our analysis in the off-shell, NWA and ET approaches are catalogued in Table 3. The key point to 


\begin{tabular}{c||c|c} 
& LO & NLO \\
\hline \hline CMS [pb] & $4.184(1)_{-12.3 \%}^{+8.5 \%}$ & $4.115(5)_{+4.6 \%}^{+0.5 \%}$ \\
\hline $\begin{array}{c}\text { NWA [pb] } \\
\% \text { diff }\end{array}$ & $\begin{array}{c}4.223(1)_{-12.2 \%}^{+8.8 \%} \\
+0.9\end{array}$ & $\begin{array}{c}4.138(1)_{+2.6 \%}^{+0.9 \%} \\
+0.6\end{array}$ \\
\hline ET [pb] & $4.154(1)_{-12.2 \%}^{+8.8 \%}$ & $4.074(1)_{+4.0 \%}^{+0.3 \%}$ \\
$\%$ diff & -0.7 & -1.0 \\
\hline
\end{tabular}

Table 3: LHC (8 TeV) cross sections for the process defined via the analysis of Table 1, at LO and NLO for the off-shell (CMS), NWA and ET computations. Numbers in brackets are Monte Carlo integration uncertainties whilst the percentages indicate scale uncertainties. '\%diff' is the $\%$ difference to the CMS results.

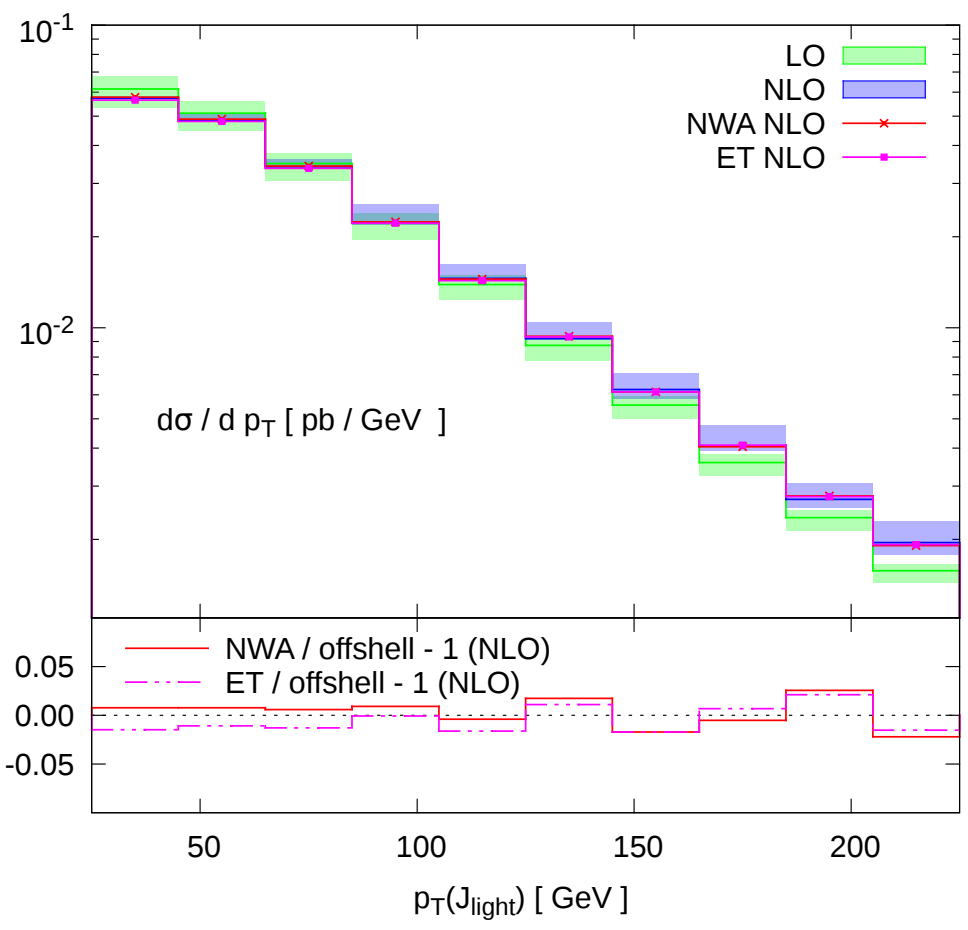

Figure 4: Transverse momentum of light jet, $p_{T}\left(J_{\text {light }}\right)$.

highlight here is the small difference, $\mathcal{O}(1-2 \%)$, between the three approaches, consistent with our expectation that it be parametrically suppressed in the NWA 
by terms of $\mathcal{O}\left(\Gamma_{t} / m_{t}\right)$ for inclusive observables. Indeed, similar small-sized differences are observed for differential observables either inclusive in, or insensitive to, the invariant mass of the $\left(W^{+}, J_{b}\right)$-system. As an illustrative example we present in Figure 4 the transverse momentum distribution of the light jet, $p_{T}\left(J_{\text {light }}\right)$. The lower panel reveals that the NWA and ET NLO results differ by $1-2 \%$ in all bins from the off-shell NLO results. In the upper panel it can be seen that both the NWA and ET results are actually contained within the scale variation band of the NLO off-shell result, indicating that for this observable the size of off-shell effects is smaller than the scale uncertainty.

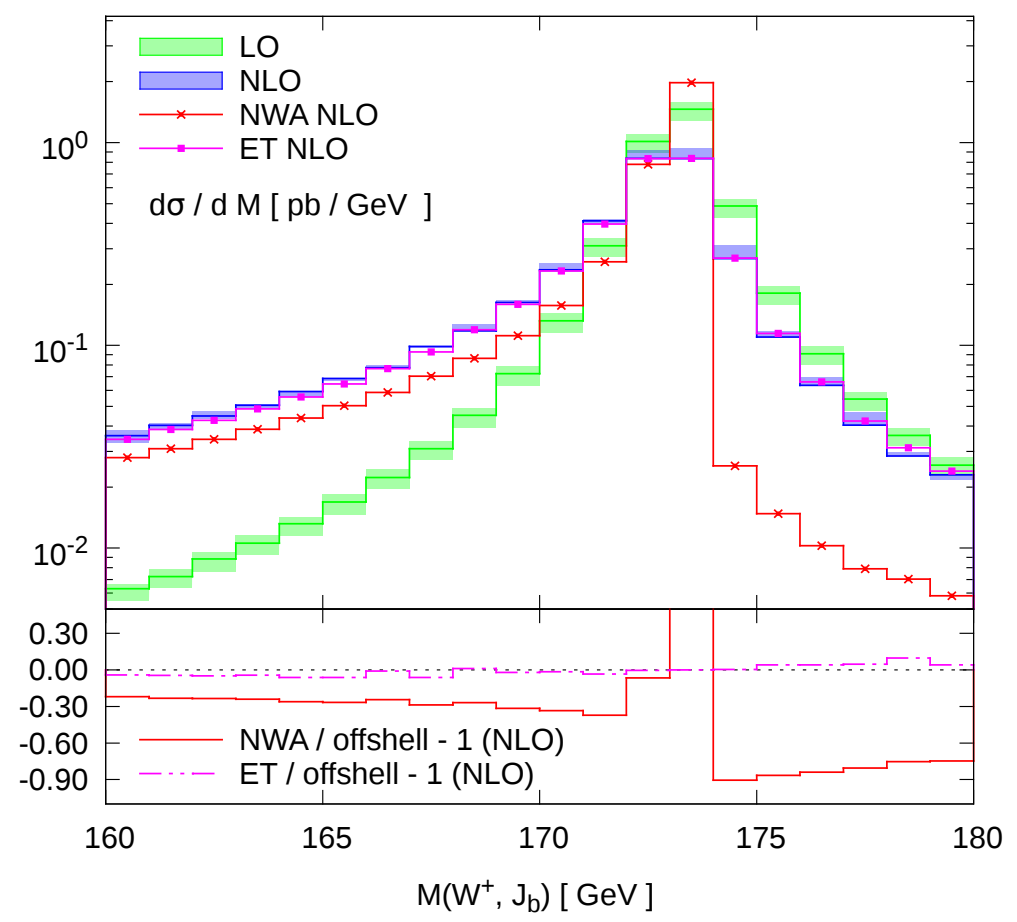

Figure 5: Invariant mass distribution for the reconstructed top quark, $M\left(W^{+}, J_{b}\right)$.

The picture changes for observables which are less inclusive in the invariant mass of the reconstructed top quark (i.e., the $\left(W^{+}, J_{b}\right)$-system), with the prime example being of course the invariant mass itself, displayed in Figure 5. The first feature one observes is that the NLO corrections are large, in particular below the peak position. The origin of these is to a large extent the real corrections to the top decay, confirmed by the fact that the NWA result mimics the shape of the off-shell curve for $M\left(W^{+}, J_{b}\right)<m_{t}$. However, it is clear that the shapes of the 
distributions predicted by the NWA and off-shell calculations are very different for $M\left(W^{+}, J_{b}\right)>m_{t}$ where the NWA curve is much steeper than the one in which finite-width effects have been included. This is due to the fact that in the NWA the $W^{+} J_{b}$ invariant mass can only receive contributions to $M\left(W^{+}, J_{b}\right)>$ $m_{t}$ from real NLO corrections, where the additional radiation originates in the production process, and is then clustered into the $b$-jet. On the contrary, in the off-shell calculation, the $M\left(W^{+}, J_{b}\right)$ distribution receives contributions in this region already at the LO, both from resonant diagrams (due to the off-shellness of the top quark), as well as from non-resonant ones. As indicated in the lower panel, near the peak of the distribution and for larger mass values the difference between the full off-shell result and that in the NWA exceeds $60 \%$ and is much bigger than scale uncertainty. Another important feature to pick out from the lower panel is the change in sign of this difference at the peak. This is the reason why, despite the sizeable differences between the off-shell and NWA distributions seen in Figure 5, the off-shellness only results in small corrections for inclusive observables [21, 22]. In contrast to the case of the NWA, the agreement between the off-shell and ET results is striking, over the full range considered here.

We now examine Figure 6, where we plot the distribution of transverse $b$-jet momentum relative to the direction of flight of the reconstructed top quark in the reconstructed top-quark rest frame, $p_{T}\left(J_{b}\right)_{\text {rel.t. }}$. At the LO and when the top quark is assumed to be on-shell this observable has a kinematic cut-off at $p_{T}\left(J_{b}\right)_{\text {rel.t }}=$ $\left(m_{t}^{2}-m_{W}^{2}\right) /\left(2 m_{t}\right)$. Once again, the difference between the NWA and off-shell approaches changes sign at the sharp edge of the distribution, and beyond it finite-width effects can be very large. Both the NWA and ET results approximate the off-shell one well in the region below the edge. Close to the peak and beyond it, the NWA is in vast disagreement with the off-shell calculation, as was the case for the invariant-mass distribution. The ET prediction gives a rather good description at the peak; beyond the edge the ET and off-shell are still relatively close to each other (i.e., almost within scale variation), but there is an obvious trend in the difference between the two. This is an indication that, towards the upper end of the range considered, a region of phase space is probed in which nonresonant, or subleading- $\Gamma_{t}$, contributions become important. For the same reason, similar differences between the ET and CMS results will show up in the tails of the invariant mass distribution should the range considered there be enlarged. Figures 5 and 6 underline that the use of such observables in certain contexts (e.g., template fitting) requires care when using NWA predictions. Finally, we stress that these findings are consistent with and complement those discussed in recent studies of finite-width effects in top quark production [21, 22, 26, 27, 28, 29]. 


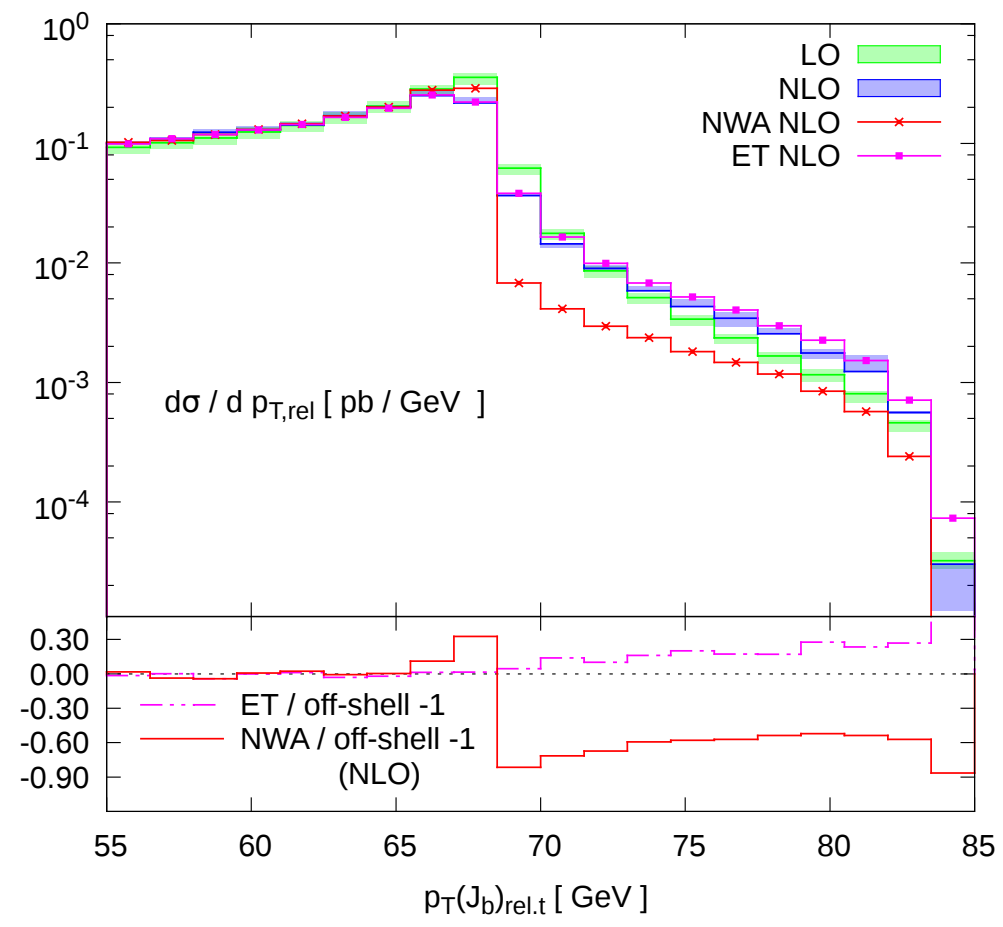

Figure 6: Transverse momentum of $b$-jet relative to flight of top quark, in reconstructed top quark rest frame, $p_{T}\left(J_{b}\right)_{\text {rel.t. }}$.

\section{Conclusions}

In this letter we have performed the computation of NLO QCD corrections to EW $t$-channel $W^{+} b j$ production. The calculation, carried out within the AMC@NLO framework, was done making use of the complex-mass scheme, and retains the full off-shell and interference effects at NLO. In addition we have compared our results with those obtained with the NWA and ET approaches. We conclude that, at least in the case of the top quark, it is incorrect to claim that the NWA is an excellent approximation universally. While the NWA gives a good description of many observables, it fails dramatically for others, in particular those sensitive to the invariant mass of the $\left(W^{+}, J_{b}\right)$-system. On the other hand, we find that the predictions of the ET approach are much closer to those of the full NLO QCD results. These two facts combined imply that for certain observables off-shell effects are much more relevant for a correct description of the final-state kinematics, than NLO corrections to the top-quark decay alone (which include hard radiation off the $b$ quark). We feel that this is a general conclusion 
and should be applicable regardless of the hard process. This has already been confirmed by similar results reported in the literature for $t \bar{t}$ production. Clearly, along with the dominant off-shell effects, there are process-dependent and beyondleading- $\Gamma_{t}$ effects, which one can start to see in the small differences between the full NLO QCD and ET results. These differences, which are numerically subleading for the present analysis, are expected to become larger if the non-resonant effects are allowed to become important, for example by requiring the invariant mass of $\left(W^{+}, J_{b}\right)$-system to be significantly larger than $m_{t}$.

An important future study will be that of assessing the impact that parton showers and hadronization have on the distributions presented here. Closely connected to this, possible systematics on the top-mass extraction introduced by ignoring finite-width effects at the matrix element level can be quantified for single-top for a realistic experimental setup.

\section{Acknowledgements}

We thank the authors of [22] for providing us with their code for the ET results. AP thanks Paolo Torrielli for his help with AMC@NLO and gratefully acknowledges the support and hospitality of the CERN Theory division where much of this work was completed. This research has been supported by the ERC grant 291377 "LHCtheory: Theoretical predictions and analyses of LHC physics:

advancing the precision frontier." SF is on leave of absence from INFN, Sez. di Genova.

\section{References}

\section{References}

[1] CDF Collaboration, T. Aaltonen et al., Phys.Rev.Lett. 103 (2009) 092002, arXiv:0903.0885 [hep-ex].

[2] Do Collaboration, V. Abazov et al., Phys.Rev.Lett. 103 (2009) 092001, arXiv:0903.0850 [hep-ex].

[3] H. Lacker, A. Menzel, F. Spettel, D. Hirschbuhl, J. Luck, et al., Eur.Phys.J. C72 (2012) 2048, arXiv:1202.4694 [hep-ph].

[4] S. S. Willenbrock and D. A. Dicus, Phys.Rev. D34 (1986) 155. 
[5] B. Harris, E. Laenen, L. Phaf, Z. Sullivan, and S. Weinzierl, Phys.Rev. D66 (2002) 054024, arXiv:hep-ph/0207055 [hep-ph].

[6] J. M. Campbell, R. Frederix, F. Maltoni, and F. Tramontano, Phys.Rev.Lett. 102 (2009) 182003, arXiv:0903.0005 [hep-ph].

[7] J. M. Campbell, R. Frederix, F. Maltoni, and F. Tramontano, JHEP 0910 (2009) 042, arXiv:0907.3933 [hep-ph].

[8] M. Beccaria, C. Carloni Calame, G. Macorini, E. Mirabella, F. Piccinini, et al., Phys.Rev. D77 (2008) 113018, arXiv:0802.1994 [hep-ph].

[9] N. Kidonakis, Phys.Rev. D81 (2010) 054028, arXiv:1001.5034 [hep-ph].

[10] N. Kidonakis, Phys.Rev. D83 (2011) 091503, arXiv:1103.2792 [hep-ph].

[11] H. X. Zhu, C. S. Li, J. Wang, and J. J. Zhang, JHEP 1102 (2011) 099, arXiv:1006.0681 [hep-ph].

[12] J. Wang, C. S. Li, H. X. Zhu, and J. J. Zhang, arXiv:1010.4509 [hep-ph].

[13] S. Frixione, E. Laenen, P. Motylinski, and B. R. Webber, JHEP 0603 (2006) 092, arXiv:hep-ph/0512250 [hep-ph].

[14] S. Alioli, P. Nason, C. Oleari, and E. Re, JHEP 0909 (2009) 111, arXiv:0907.4076 [hep-ph].

[15] R. Frederix, E. Re, and P. Torrielli, JHEP 1209 (2012) 130, arXiv:1207.5391 [hep-ph].

[16] J. M. Campbell, R. K. Ellis, and F. Tramontano, Phys.Rev. D70 (2004) 094012, arXiv:hep-ph/0408158 [hep-ph].

[17] S. Heim, Q.-H. Cao, R. Schwienhorst, and C.-P. Yuan, Phys.Rev. D81 (2010) 034005, arXiv:0911.0620 [hep-ph].

[18] R. Schwienhorst, C.-P. Yuan, C. Mueller, and Q.-H. Cao, Phys.Rev. D83 (2011) 034019, arXiv:1012.5132 [hep-ph].

[19] J. van der Heide, E. Laenen, L. Phaf, and S. Weinzierl, Phys.Rev. D62 (2000) 074025, arXiv:hep-ph/0003318 [hep-ph]. 
[20] R. Pittau, Phys.Lett. B386 (1996) 397-402, arXiv:hep-ph/9603265 [hep-ph].

[21] P. Falgari, F. Giannuzzi, P. Mellor, and A. Signer, Phys.Rev. D83 (2011) 094013, arXiv:1102.5267 [hep-ph].

[22] P. Falgari, P. Mellor, and A. Signer, Phys.Rev. D82 (2010) 054028, arXiv:1007.0893 [hep-ph].

[23] V. S. Fadin, V. A. Khoze, and A. D. Martin, Phys.Rev. D49 (1994) 2247-2256.

[24] V. S. Fadin, V. A. Khoze, and A. D. Martin, Phys.Lett. B320 (1994) 141-144, arXiv:hep-ph/9309234 [hep-ph].

[25] K. Melnikov and O. I. Yakovlev, Phys.Lett. B324 (1994) 217-223, arXiv:hep-ph/9302311 [hep-ph].

[26] G. Bevilacqua, M. Czakon, A. van Hameren, C. G. Papadopoulos, and M. Worek, JHEP 1102 (2011) 083, arXiv:1012.4230 [hep-ph].

[27] A. Denner, S. Dittmaier, S. Kallweit, and S. Pozzorini, JHEP 1210 (2012) 110, arXiv:1207.5018 [hep-ph].

[28] A. Denner, S. Dittmaier, S. Kallweit, and S. Pozzorini, Phys.Rev.Lett. 106 (2011) 052001, arXiv:1012.3975 [hep-ph].

[29] P. Falgari, A. Papanastasiou, and A. Signer, arXiv:1303.5299 [hep-ph].

[30] K. Melnikov and M. Schulze, JHEP 0908 (2009) 049, arXiv:0907.3090 [hep-ph].

[31] J. M. Campbell and R. K. Ellis, arXiv:1204.1513 [hep-ph].

[32] R. G. Stuart, Phys.Lett. B272 (1991) 353-358.

[33] A. Aeppli, G. J. van Oldenborgh, and D. Wyler, Nucl.Phys. B428 (1994) 126-146, arXiv:hep-ph/9312212 [hep-ph] .

[34] A. Denner, S. Dittmaier, M. Roth, and D. Wackeroth, Nucl.Phys. B560 (1999) 33-65, arXiv:hep-ph/9904472 [hep-ph]. 
[35] A. Denner, S. Dittmaier, M. Roth, and L. Wieders, Nucl.Phys. B724 (2005) 247-294, arXiv:hep-ph/0505042 [hep-ph].

[36] D. Buarque Franzosi, V. Hirschi, and O. Mattelaer, in preparation .

[37] V. Hirschi, R. Frederix, S. Frixione, M. V. Garzelli, F. Maltoni, et al., JHEP 1105 (2011) 044, arXiv:1103.0621 [hep-ph].

[38] R. Frederix, S. Frixione, F. Maltoni, and T. Stelzer, JHEP 0910 (2009) 003, arXiv:0908.4272 [hep-ph].

[39] SM AND NLO MULTILEG and SM MC Working Groups, J. Alcaraz Maestre et al., arXiv:1203.6803 [hep-ph].

[40] W. Bernreuther, A. Brandenburg, Z. Si, and P. Uwer, Nucl.Phys. B690 (2004) 81-137, arXiv:hep-ph/0403035 [hep-ph].

[41] A. Martin, W. Stirling, R. Thorne, and G. Watt, Eur.Phys.J. C63 (2009) 189-285, arXiv:0901.0002 [hep-ph].

[42] S. Catani, Y. L. Dokshitzer, M. Seymour, and B. Webber, Nucl.Phys. B406 (1993) 187-224.

[43] S. D. Ellis and D. E. Soper, Phys.Rev. D48 (1993) 3160-3166, arXiv:hep-ph/9305266 [hep-ph].

[44] M. Cacciari, G. P. Salam, and G. Soyez, Eur.Phys.J. C72 (2012) 1896, arXiv:1111.6097 [hep-ph]. 